\title{
Creating and evaluating replicas of surfaces machined by laser beam
}

\author{
Milena Kubišová ${ }^{1, *}$, Vladimir Pata ${ }^{1}$, and Libuše Sýkorová ${ }^{1}$ \\ ${ }^{1}$ Tomas Bata University in Zlín, Faculty of Technology, Vavrečkova 275, 76001 Zlín, Czech \\ Republic
}

\begin{abstract}
In industrial practice, we often encounter a problem when it is necessary to measure and subsequently evaluate the surface quality of the part produced by the water jet, laser cutting. In that case, it is very often difficult to evaluate the final surface because of its weight or bulkiness. However, the literature sources recommend using of material known under the commercial name Dentacryl ${ }^{\mathrm{TM}}$. Although this material is still being used in technical practice, its utilisation for creating the replicas of the polypropylene surfaces which is produced by the laser beam is not recommended due to its limited viscosity and mainly due to its health risk for allergic people. Therefore, this article will describe a method of an application the two-component dental material Siloflex ${ }^{\mathcal{O}}$ for the creation of the replicas of polypropylene surfaces formed after $\mathrm{CO}_{2}$ laser machining. Furthermore, the statistical approach for evaluation of the quality comparison of the original surfaces and produced replicas is necessary to surface quality of produced replicas.
\end{abstract}

\section{Introduction}

The main part of this article is the research of the preparation of replicas of surfaces and the determination of the methods impression and its evaluation. This special technology is very little explored from the surface quality control of products in which can brings new trends and methods of evaluation mostly in the section of the production of the moulds. For creating replicas of surfaces is historically the most commonly used the material known as Dentacryl $^{\mathrm{TM}}$. In the nowadays modern concept of the quality, assessment is this material problematic inapplicable terms of both mechanical as well as security reasons.

For the production of replicas, we use the impression compounds, mainly used in dentistry. In this field, we can search not only the inspiration for the choice of material suitable for scanning surfaces but also knowledge in the preparation and the application itself.

Another independent issue consists of the discovery of suitable dental impression materials of the required properties (as discussed above) is followed to discover suitable mathematical and statistical methods to the assessment of the quality of the replicas.

\footnotetext{
*Corresponding author: mkubisova@,ft.utb.cz
} 
The proposed article will deal with a method of the production of the replicas using the dental materials SILOFLEX ${ }^{\odot}$ including the technological process of the replicas. It will propose methods based on the statistical hypothesis testing to assess the degree of the agreement between the original surface and the replica. The scanning method of these two surfaces the original and the replica will be performed by using the contactless manner matrix CLI method [1].

\section{Materials and Methods}

\subsection{Machining of Polypropylene Surfaces by CO2 laser}

When creating the model surfaces on polypropylene (from now on referred to as PP) plates, the $\mathrm{CO}_{2}$ laser ILS 3NM with the following characteristics was used: the maximum power of $100 \mathrm{~W}$, the maximum feed rate of $1524 \mathrm{~mm} \cdot \mathrm{s}^{-1}$ [8]. Next, the parameters of laser power and feed were changed - and Tab.1.

Table 1. Machining parameters.

\begin{tabular}{|c|c|c|c|c|}
\hline $\begin{array}{l}\mathrm{P}[\mathrm{W}] \\
\mathrm{f}\left[\mathrm{mm} \cdot \mathrm{s}^{-1}\right]\end{array}$ & 1. & 2. & 3. & 4. \\
\hline 1. & $20 / 305$ & $20 / 610$ & $20 / 915$ & $20 / 1220$ \\
\hline 2. & $40 / 305$ & $40 / 610$ & $40 / 915$ & $40 / 1220$ \\
\hline 3. & $60 / 305$ & $40 / 610$ & $60 / 915$ & $60 / 1220$ \\
\hline 4. & $80 / 305$ & $80 / 610$ & $80 / 915$ & $80 / 1220$ \\
\hline
\end{tabular}

\subsection{Replica Creation}

From all surfaces that were produced under cutting conditions (see Table 1), were formed the replicas using the dental material Siloflex ${ }^{\circ}$.

As a typical case used for the purpose of this article was choose the surface made at the cutting conditions $\mathrm{P}=20 \mathrm{~W}$ and $\mathrm{f}=1220 \mathrm{~mm}$.s -1 (see Table1). The reason for choosing this sample was that the final surface showed maximum relief section and due to the biggest problems with separability of the replica from the sample. The last surface of the product and its replica (Fig. 2) is characterised by many surface defects, such as sinks, these defects complicate the replica production, scanning method, filtration type and the subsequent statistical evaluation [2].

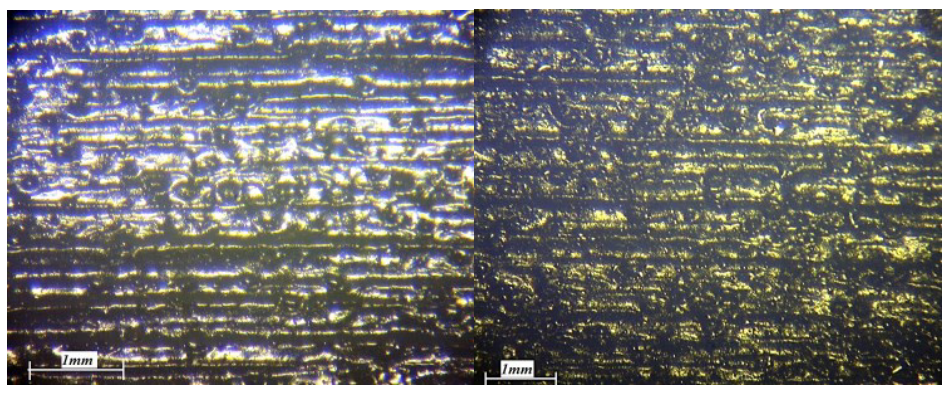

A.

B.

Fig. 1. MS 


\subsection{Evaluation of the method}

Firstly, original surfaces of dimensions $5 \times 5 \mathrm{~mm}$ were scanned using 3D surface scanner Talysurf CLI 500 with a resolution of $0,25 \mu \mathrm{m}$ in the $\mathrm{X}$ and Y axes. Sensitivity in Z axis was allowed to a maximum possible level $1 \mathrm{~nm}$.

After scanning the individual values of $\mathrm{X}, \mathrm{Y}$, and $\mathrm{Z}$ parameters into a three-dimensional data matrix. The data was filtrated using a double Gaussian sturdy filter to ensure the correct calculation of amplitude parameters of surface quality of the originals and the replicas.
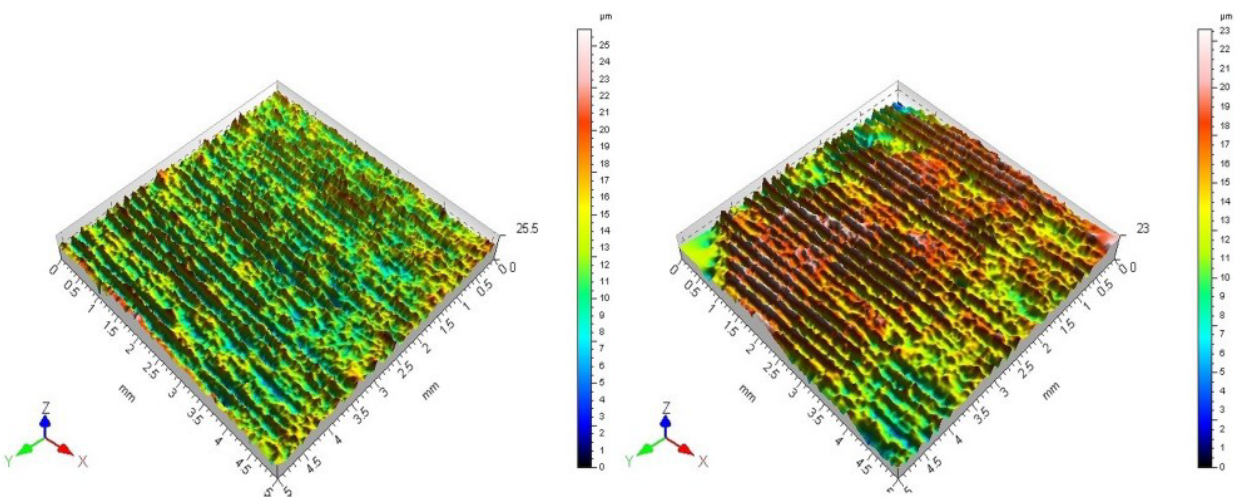

Fig. 2. 3D scanned: A) original B) replica under conditions: PPI 1000, P $20 \%$ and $\mathrm{f} 80 \%$.

To specify whether the original and the replica are close, each to the other ISO 4287 was applied. We look at main parameters: Arithmetic mean deviation of the assessed profile $\mathrm{Ra}$ and a maximum height of profile $\mathrm{Rz}$ [3-4].

Table 2. Roughness parameters by ISO 4287 for original and replica

\begin{tabular}{|c|c|c|c|c|c|c|}
\hline \multicolumn{2}{|c|}{ Original } & Mean & Std. Dev. & Median & Min & Max \\
\hline $\mathrm{Rp}$ & $\mu \mathrm{m}$ & 4,35 & 0,64 & 4,40 & 2,70 & 6,09 \\
\hline $\mathrm{Rv}$ & $\mu \mathrm{m}$ & 4,44 & 0,74 & 4,44 & 2,82 & 6,17 \\
\hline $\mathrm{Rz}$ & $\mu \mathrm{m}$ & 8,79 & 1,29 & 8,88 & 6,10 & 12,10 \\
\hline $\mathrm{Rt}$ & $\mu \mathrm{m}$ & 11,70 & 1,81 & 11,80 & 7,31 & 16,10 \\
\hline $\mathrm{Ra}$ & $\mu \mathrm{m}$ & 1,87 & 0,29 & 1,87 & 1,21 & 2,53 \\
\hline
\end{tabular}

\begin{tabular}{|l|c|c|c|c|c|c|}
\cline { 2 - 7 } \multicolumn{2}{l|}{ Replica } & Mean & Std. Dev. & Median & Min & Max \\
\hline Rp & $\mu \mathrm{m}$ & 3,93 & 0,792 & 3,91 & 2,56 & 6,44 \\
\hline $\mathrm{Rv}$ & $\mu \mathrm{m}$ & 4,05 & 0,734 & 4,13 & 2,52 & 5,62 \\
\hline $\mathrm{Rz}$ & $\mu \mathrm{m}$ & 7,97 & 1,450 & 7,960 & 5,12 & 11,4 \\
\hline $\mathrm{Rt}$ & $\mu \mathrm{m}$ & 10,5 & 2,070 & 10,30 & 6,64 & 16,4 \\
\hline $\mathrm{Ra}$ & $\mu \mathrm{m}$ & 1,67 & 0,269 & 1,73 & 1,01 & 2,25 \\
\hline
\end{tabular}

After inventing methods, impression replicas were needed to prove that they can originate with a replica similar. For this demonstration was ingested to ISO 4287.

From the table 2, it is clear that the surface of the original and a replica are very similar. The difference between the original and the replica was calculated for parameters $\mathrm{Ra} 0,2$ $\mu \mathrm{m}$ and $\mathrm{Rz} 0,82 \mu \mathrm{m}$. Evaluation of the degree of similarity based on cluster analysis [5-7].

\subsection{Evaluation of the degree of similarity based on ,, Cluster analysis.”}

In the case of cluster analysis was as the optimal method chosen Ward's method, linking was the Euclidean distance.

It was found that the measured values disintegrate into two clusters, namely amplitude parameters of the originals and replicas see figure 3 and table 3. 


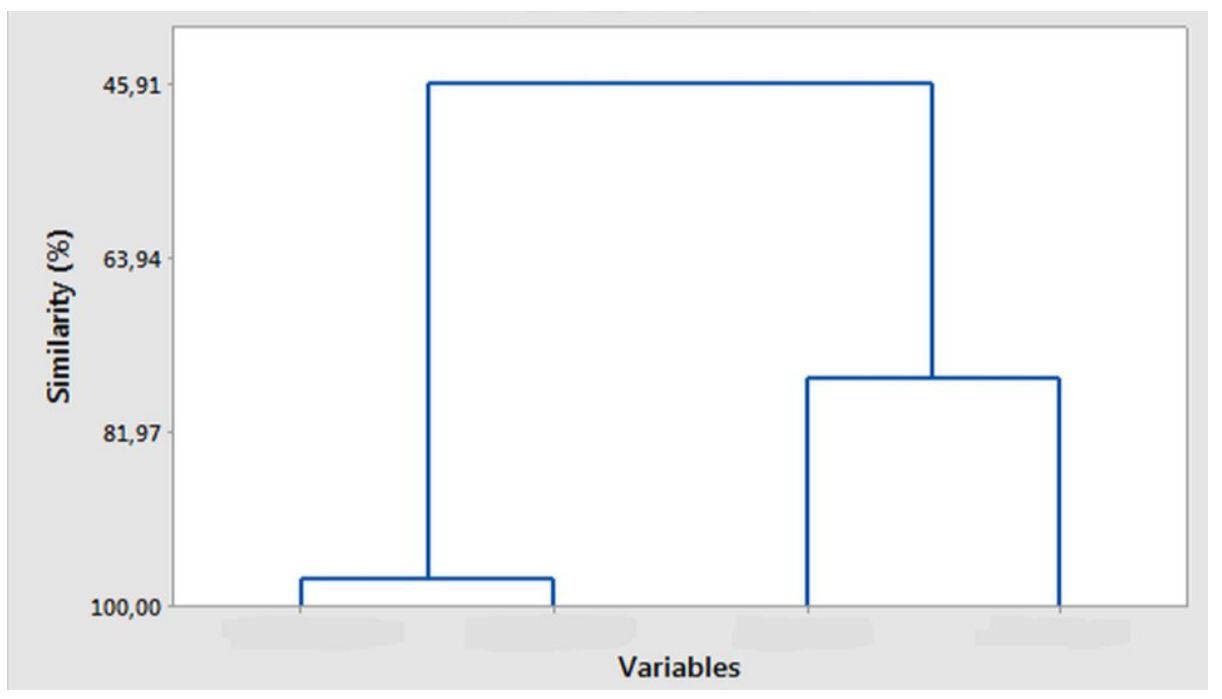

Fig. 3. Graph Cluster analysis

Table 3. Numerical results of Cluster analysis

\begin{tabular}{|c|c|c|l|}
\hline Step & $\begin{array}{c}\text { Number of } \\
\text { clusters }\end{array}$ & $\begin{array}{c}\text { Similarity } \\
\text { level }\end{array}$ & \multicolumn{1}{|c|}{ Cluster } \\
\hline 1 & 3 & 97,2496 & Rz_Original Ra_Original \\
\hline 2 & 2 & 76,3666 & Rz_Replica Ra_Replica \\
\hline 3 & 1 & 45,9051 & $\begin{array}{l}\text { Rz_Original Ra_Original and Rz_Replica } \\
\text { Ra_Replica }\end{array}$ \\
\hline
\end{tabular}

To confirm the obtained results, Factor Analysis which has been optimised for the two main factors Varimax methodology were further applied. Biplot diagram (see Fig. 4), which again clearly demonstrates significant agreement from the above-displayed Cluster analysis, was selected as a typical demonstrates [2].

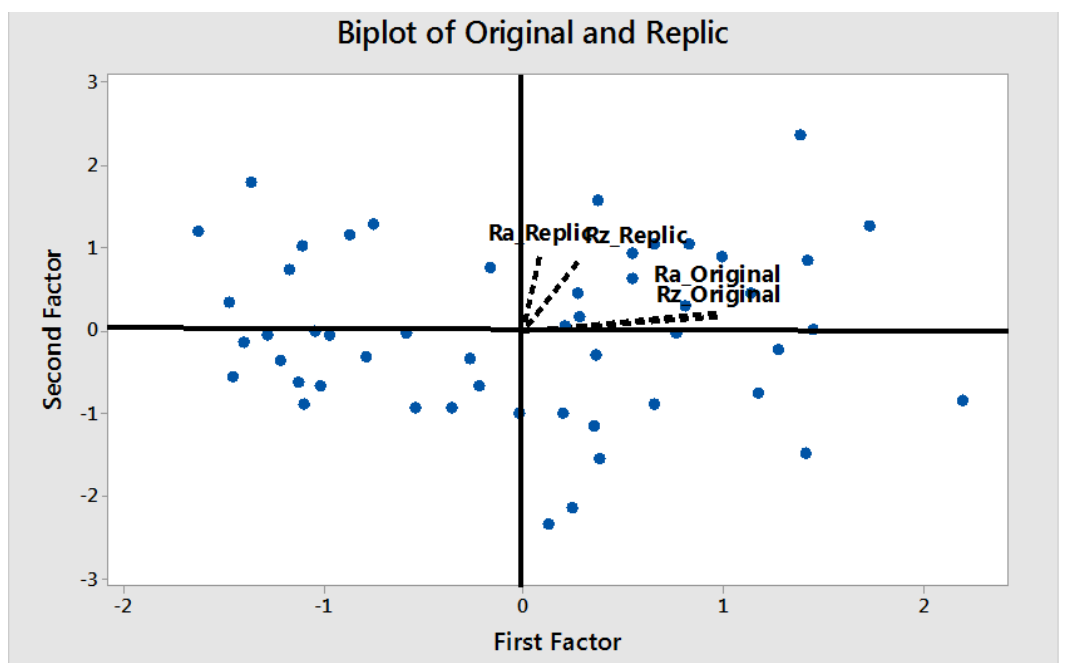

Fig. 4. Biplot diagram 
Table 4. Unroasted Factor Loadings and Communalities

\begin{tabular}{|l|c|c|c|c|c|}
\hline Variable & Factor 1 & Factor 2 & Factor 3 & Factor 4 & Communality \\
\hline Rz_Original & 0.879 & -0.445 & -0.055 & 0.162 & 1 \\
\hline Ra_Original & 0.901 & -0.397 & -0.052 & -0.166 & 1 \\
\hline Rz_Replica & 0.703 & 0.498 & 0.508 & 0.005 & 1 \\
\hline Ra_Replica & 0.591 & 0.675 & -0.442 & 0.005 & 1 \\
\hline Variance & 2.820 & 1.059 & 0.459 & 0.054 & 4 \\
\hline \% Var & 0.607 & 0.265 & 0.115 & 0.013 & 1 \\
\hline
\end{tabular}

Table 5. Unroasted Factor Loadings and Communalities Varimax Rotation

\begin{tabular}{|l|c|c|c|c|c|}
\hline Variable & Factor 1 & Factor 2 & Factor 3 & Factor 4 & Communality \\
\hline Rz_Original & 0.972 & 0.092 & 0.151 & 0.157 & 1 \\
\hline Ra_Original & 0.959 & 0.128 & 0.184 & -0.171 & 1 \\
\hline Rz_Replica & 0.208 & 0.273 & 0.939 & -0.003 & 1 \\
\hline Ra_Replica & 0.122 & 0.959 & 0.256 & -0.003 & 1 \\
\hline Variance & 1.270 & 1.019 & 1.005 & 0.054 & 4 \\
\hline \% Var & 0.481 & 0.255 & 0.251 & 0.013 & 1 \\
\hline
\end{tabular}

Table 6. Factor Score Coefficients

\begin{tabular}{|l|l|l|l|c|}
\hline Variable & Factor 1 & Factor 2 & Factor 3 & Factor 4 \\
\hline Rz_Original & 0.561 & -0.034 & -0.105 & 3.015 \\
\hline Ra_Original & 0.521 & -0.046 & -0.111 & -3.087 \\
\hline Rz_Replica & -0.172 & -0.295 & 1.189 & 0.094 \\
\hline Ra_Replica & -0.074 & 1.136 & -0.313 & 0.097 \\
\hline
\end{tabular}

\section{Conclusion}

That article describes the ability of a particular method of using dental silicone materials Siloflex ${ }^{\odot}$ to produce replicas of removable difficult surfaces on which should be evaluated their quality.

For this purpose, is given by accurate procedure silicone mass in the manufacture of surface replicas and is applied the methodology of scanning the surface itself and the surface of the replicas using contactless scanner Taylor Hobson CLI 500.

This is followed by recommendations amplitude comparison of selected surface parameters using statistical methods multidimensional, namely Cluster analysis, Factor analysis using Varimax technique.

Finally, it can be stated that by comparing the amplitude parameters of similarities, their differences do not exceed $7 \%$, for the purpose of laser machining surface material polypropylene can be considered the satisfactory result.

Acknowledgements: This work and the project is realised with the financial support of the internal grant of TBU in Zlín No. IGA/FT/2017/002 funded from the resources of specific university research.

\section{References}

1. B. Muralikrishnan, J. Raja, Computational surface and roundness metrology (Springer, New York, 2009)

2. M. Bilodeau, D. Brenner, Theory of multivariate statistics (Springer, New York, 1999)

3. Intelligent Laser System III-NM - Operation Manual, Version 1.(Laser Tools \& Technics Corp., 2007) 
4. R.D. Reiss, M. Thomas, Statistical analysis of extreme values: with applications to insurance, finance, hydrology and other fields (Birkhäuser Verlag, 2nd ed, Basel, 2001)

5. R. Stasiak-Betlejewsk, A. Czajkowska, MATEC Web of Conferences (2017)

6. V. Diţu, G. Oancea, R. Daicu, MATEC Web of Conferences (2017)

7. A.Mat Deris, A. Mohd Zain, R. Sallehuddin, S. Sharif, MATEC Web of Conferences (2016)

8. Yang, T. and Bellouard, Y., MATEC Web of Conferences (2015) 